\title{
Unsaturated Phytosterol
}

National Cancer Institute

\section{Source}

National Cancer Institute. Unsaturated Phytosterol. NCI Thesaurus. Code C68437.

Any steroid derivative bearing unsaturated bonds, typically found in position 5-6 of the B ring of the steroid skeleton. 